\title{
Results of bent crystal channeling and collimation at the Relativistic Heavy Ion Collider
}

\author{
R. P. Fliller III, ${ }^{1, *}$ A. Drees, ${ }^{2}$ D. Gassner, ${ }^{2}$ L. Hammons, ${ }^{2}$ G. McIntyre, ${ }^{2}$ S. Peggs, ${ }^{2}$ D. Trbojevic, ${ }^{2}$ V. Biryukov, ${ }^{3}$ \\ Y. Chesnokov, ${ }^{3}$ and V. Terekhov ${ }^{3}$ \\ ${ }^{1}$ Department of Physics and Astronomy, University of Stony Brook, Stony Brook, New York, 11794, USA \\ ${ }^{2}$ Brookhaven National Laboratory, Upton, New York 11973, USA \\ ${ }^{3}$ Institute for High Energy Physics, 142281 Protvino, Russia
}

(Received 16 September 2005; published 20 January 2006)

\begin{abstract}
Bent crystal channeling has been observed with protons and fully stripped gold ions in the Relativistic Heavy Ion Collider (RHIC). Prior to 2003, a bent crystal was installed in one ring of RHIC as the first stage of a two stage collimation system. The observed channeling efficiency was approximately $25 \%$, less than half of original predictions. We show that this is due to a difference between the model and real Twiss parameters at the crystal location and our improved understanding of the beam halo. Collimation using the crystal was unsuccessful and raised background at the STAR detector by as much as a factor of 2 because of the low channeling efficiency. We give a report of our channeling studies in RHIC and describe our experience using the bent crystal as a collimator. The results are discussed and compared to simulations and theoretical predictions.
\end{abstract}

DOI: 10.1103/PhysRevSTAB.9.013501

PACS numbers: 41.85.Si, 29.20.Dh, 29.27.-a

\section{INTRODUCTION}

The Relativistic Heavy Ion Collider (RHIC) consists of two $3833 \mathrm{~m}$ superconducting rings which are capable of accelerating a range of ion species from polarized protons to fully stripped gold ions. RHIC is designed so that collisions involving different ion species are possible, for example, the deuteron gold run in 2003. Five nuclear physics experiments reside at four of the six interaction regions.

Beam losses are becoming an increasing concern for modern accelerators causing equipment damage, residual radiation, and large experimental backgrounds. In high energy colliders, such as the LHC, the stored energy in the beams is large enough that a small fraction of the beam deposited into a dipole magnet can destroy it [1]. But even at RHIC, an efficient collimation system is needed to remove the tails of the beam, minimize distributed losses, and reduce background in experiments.

Slow beam losses are caused by particles on unstable orbits forming the beam halo, or tail. Usually, multiple sets of jaws are used to remove the beam halo. An initial set, called the primary jaws, intercepts particles with small impact parameters. These particles scatter forming a secondary halo. A second set of jaws is used to intercept this secondary halo to restrict its spread and increase the collimation efficiency [2]. However, the large range of scattering angles from the primary jaws makes optimizing the location of the secondary jaws a difficult task and limits the achievable collimation efficiency.

\footnotetext{
*Email address: fliller@ fnal.gov Present address: Fermi National Accelerator Laboratory, Batavia, IL 60510, USA.
}

It is possible to use bent crystal channeling to deflect particle beams [3-7]. If the primary collimator is replaced with a bent crystal, it will be possible to deflect halo particles away from the beam core. The secondary jaws can be efficiently placed to intercept the channeled beam. If the bent crystal has a high channeling efficiency and the beam is matched to the crystal acceptance, the efficiency of the collimation system can be greatly improved over a conventional two stage collimation system.

The idea to use a bent crystal for collimation in RHIC was first proposed in 1997 [8]. A bent crystal collimator was installed in the counterclockwise ring of RHIC prior to the 2001 run. In this paper, we discuss the performance of the bent crystal as a channeling device. We present an analytical model that can be used to estimate the channeling efficiency of the bent crystal collimator. Monte Carlo simulations of multiple turn channeling are discussed. These are then compared to crystal channeling measurements. We also discuss the performance of the crystal as the first stage of a two stage collimation system.

\section{THEORY}

Crystal channeling is a phenomenon in which the crystal lattice is used to guide ions through a crystal. Lindhard was the first to provide an analytical theory of particle channeling in 1965 [9]. Ions impinging on a crystal lattice that have small angles relative to the crystal planes scatter from the lattice atoms in such a way that they follow the crystal planes. Another view is that particles see a potential between the crystal planes, $U(X)$ where $X$ is the transverse position in the plane, with $X=0$ defined as the center of the plane. The transverse kinetic energy of the particle cannot be more than the maximum value of the interplanar potential. This is known as the critical energy, $E_{c}$. For ions 
channeling in the (110) planes of silicon, the critical energy is $E_{c}=Z_{\text {ion }} 16 \mathrm{eV}$ where $Z_{\text {ion }}$ is the charge state of the impinging ion.

Assuming that the angle relative to the plane is small, the transverse kinetic energy is nonrelativistic. The critical angle is then defined as the angle of a particle whose transverse kinetic energy equals the critical energy. The critical angle is given by

$$
\theta_{c}=\sqrt{\frac{2 E_{c}}{p v}}
$$

where $p v$ is the relativistic momentum times velocity. Particles with $\theta>\theta_{c}$ will not be channeled. For silicon crystals with fully stripped gold at $100 \mathrm{GeV} / u$ or protons at $250 \mathrm{GeV}$, the critical angle is $11 \mu \mathrm{rad}$. For protons at $100 \mathrm{GeV}$, the critical angle is $19 \mu \mathrm{rad}$. A more complete theory of crystal channeling has been presented in various publications [9-11].

The crystal acceptance, A, is defined as the fraction of particles striking the crystal that can channel. Assuming that $U(X)$ is approximately harmonic, the acceptance for a straight crystal and a uniform beam with divergence $2 \Phi \geq$ $2 \theta_{c}$ is

$$
A=\frac{2 X_{c}}{d_{p}} \frac{\pi}{4} \frac{\theta_{c}}{\Phi} \approx 0.66 \frac{\theta_{c}}{\Phi},
$$

where $d_{p}$ is the distance between the crystal planes, and $X_{c}$ is defined by $U\left(X_{c}\right)=E_{c}$ [10]. The crystal acceptance equals the channeling efficiency in cases where scattering (dechanneling) in the crystal can be neglected, such as with thin crystals and high energy beams. Centrifugal terms in the interplanar potential of a curved crystal reduce the channeling efficiency by approximately $4 \%$.

Equation (2) shows that a large channeling efficiency requires that the beam striking the crystal have a small divergence. We have developed a model to estimate the angle and divergence of the beam striking the crystal. We first postulate a transverse beam distribution

$$
\rho(J, \delta)=\frac{1}{\sqrt{2 \pi \sigma_{p}} \epsilon} \exp \left[-\frac{J}{\epsilon}\right] \exp \left[-\frac{\delta^{2}}{2 \sigma_{p}^{2}}\right],
$$

where $\epsilon$ is the rms unnormalized beam emittance and $\sigma_{p}$ is the rms fractional momentum spread, $\delta$ is the fractional momentum deviation, and $J$ is the invariant of the transverse motion including dispersion,

$$
J=\frac{1}{2 \beta}\left\{(x-D \delta)^{2}+\left[x \alpha+x^{\prime} \beta-\left(D \alpha+D^{\prime} \beta\right) \delta\right]^{2}\right\}
$$

where $\left\{x, x^{\prime}\right\}$ are the transverse position and angle, $\{\beta, \alpha\}$ are the Twiss parameters, and $\left\{D, D^{\prime}\right\}$ are the dispersion and its slope. Transforming from $\{J, \delta\}$ to $\left\{x, x^{\prime}, \delta\right\}$ and integrating over all momenta produces a beam distribution

$$
\rho\left(x, x^{\prime}\right)=\frac{\exp \left[-\frac{x^{\prime 2}\left(\beta \epsilon+D^{2} \sigma_{p}{ }^{2}\right)-2 x x^{\prime}\left(-\alpha \epsilon+D D^{\prime} \sigma_{p}{ }^{2}\right)+x^{2}\left(\gamma \epsilon+D^{\prime 2} \sigma_{p}^{2}\right)}{2 \epsilon \sigma_{p}^{2}\left[\gamma D^{2}+2 \alpha D D^{\prime}+\beta D^{\prime 2}+\epsilon / \sigma_{p}{ }^{2}\right]}\right]}{2 \pi \sigma_{p} \sqrt{\epsilon\left(\gamma D^{2}+2 \alpha D D^{\prime}+\beta D^{\prime 2}+\epsilon / \sigma_{p}^{2}\right)}} .
$$

It is convenient to calculate the standard deviations of this distribution [12]

$$
\begin{aligned}
\sigma_{x}^{2} & =\epsilon \beta+D^{2}{\sigma_{p}}^{2}, \\
\sigma_{x^{\prime}}^{2} & =\gamma \epsilon+D^{\prime 2} \sigma_{p}^{2}, \\
\sigma_{x x^{\prime}} & =-\alpha \epsilon+D D^{\prime} \sigma_{p}^{2},
\end{aligned}
$$

which are, respectively, the mean square beam size, mean square angular divergence, and correlation at the location of the crystal collimator. Figure 1 shows $\rho\left(x, x^{\prime}\right)$ for the model Twiss parameters at the crystal collimator for $\beta_{\text {PHENIX }}^{*}=2 \mathrm{~m}$.

Assuming that the crystal is located a distance $x_{0}$ from the beam core and the beam strikes over the entire crystal face of width $\Delta x$, as shown in Fig. 2, the angular distribution of ions striking the crystal is given by the conditional probability distribution [12]

$$
\rho\left(x^{\prime} \mid x_{0}\right)=\frac{\int_{x_{0}}^{x_{0}+\Delta x} \rho\left(x, x^{\prime}\right) d x}{\int_{x_{0}}^{x_{0}+\Delta x} d x \int_{-\infty}^{\infty} \rho\left(x, x^{\prime}\right) d x^{\prime}} .
$$

The mean angle of particles hitting the crystal is

$$
x_{p}^{\prime}=\sqrt{\frac{2}{\pi}} \frac{\sigma_{x x^{\prime}}}{\sigma_{x}} \frac{\left[1-\exp \left(\frac{\Delta x\left(2 x_{0}+\Delta x\right)}{2 \sigma_{x}^{2}}\right)\right] \exp \left(-\frac{\left(x_{0}+\Delta x\right)^{2}}{2 \sigma_{x}^{2}}\right)}{\operatorname{erf}\left[\frac{x_{0}}{\sqrt{2} \sigma_{x}}\right]-\operatorname{erf}\left[\frac{x_{0}+\Delta x}{\sqrt{2} \sigma_{x}}\right]} .
$$

where erf is the error function. The crystal is said to be

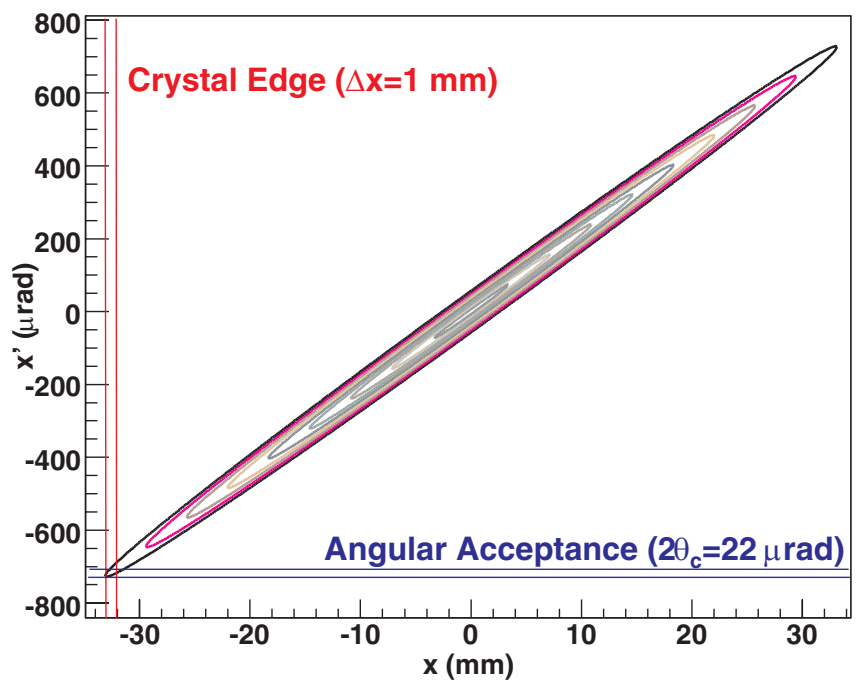

FIG. 1. (Color) Beam horizontal phase space at the RHIC crystal collimator for $\beta^{*}=2 \mathrm{~m}$ at PHENIX interaction region. Each contour is $1 \sigma$ of the beam size assuming a $15 \pi \mathrm{mmmrad}$ emittance. 


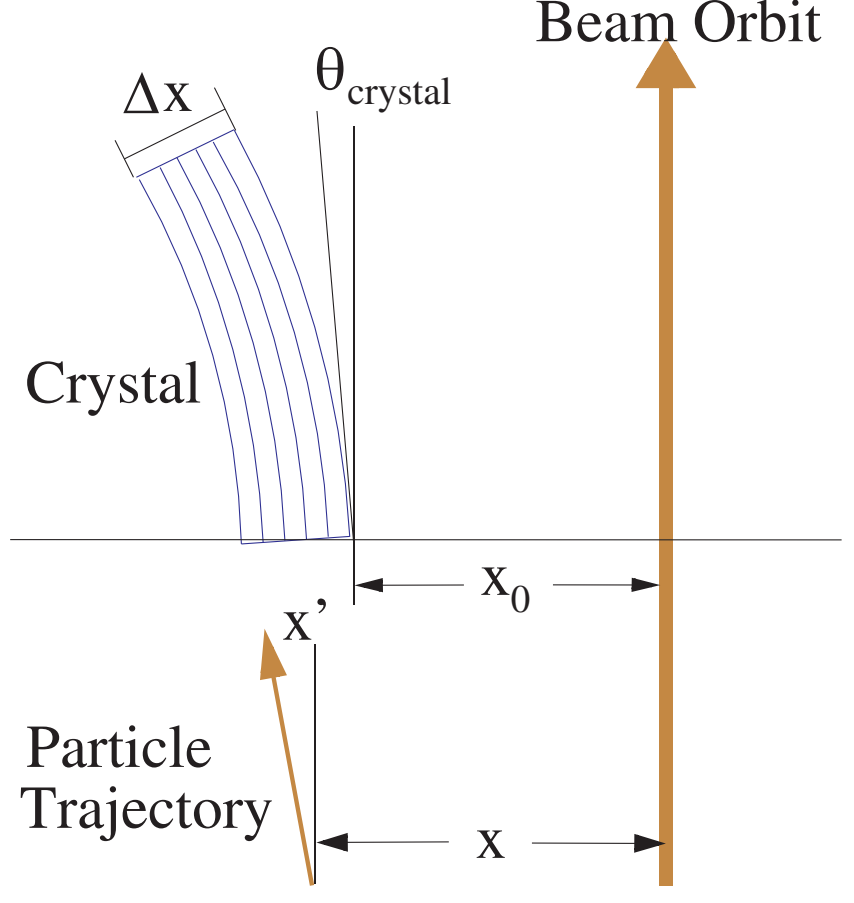

FIG. 2. (Color) Geometry used to calculate the crystal acceptance.

properly aligned if the angle of the crystal planes have an angle of $x_{p}^{\prime}$ with respect to the beam orbit.

The divergence of the beam striking the crystal is given by the rms of $\rho\left(x^{\prime} \mid x_{0}\right)$,

$$
\begin{aligned}
\sigma_{x^{\prime}}\left(x_{0}\right)= & \left\{\left[\sigma_{x^{\prime}}^{2} \exp \left[\frac{\left(x_{0}+\Delta x\right)^{2}}{2 \sigma_{x}^{2}}\right]+\sqrt{\frac{2}{\pi}} \frac{\sigma_{x x^{\prime}}^{2}}{\sigma_{x}^{2}}\right.\right. \\
& \left.\times \frac{\Delta x+x_{0}\left[1-\exp \left(\frac{\Delta x\left(2 x_{0}+\Delta x\right)}{2 \sigma_{x}{ }^{2}}\right)\right]}{\sigma_{x}\left[\operatorname{erf}\left(\frac{x_{0}}{\sqrt{2} \sigma_{x}}\right)-\operatorname{erf}\left(\frac{x_{0}+\Delta x}{\sqrt{2} \sigma_{x}}\right)\right]}\right] \\
& \times \exp \left[\frac{\left(x_{0}+\Delta x\right)^{2}}{2 \sigma_{x}^{2}}\right]-\frac{2}{\pi} \frac{\sigma_{x x^{\prime}}{ }^{2}}{\sigma_{x}{ }^{2}} \\
& \times \frac{\left[1-\exp \left(\frac{\Delta x\left(2 x_{0}+\Delta x\right)}{2 \sigma_{x}{ }^{2}}\right)\right]^{2}}{\left.\left[\operatorname{erf}\left(\frac{x_{0}}{\sqrt{2} \sigma_{x}}\right)-\operatorname{erf}\left(\frac{x_{0}+\Delta x}{\sqrt{2} \sigma_{x}}\right)\right]^{2}\right\}^{1 / 2}} \\
& \times \exp \left[-\frac{\left(x_{0}+\Delta x\right)^{2}}{2 \sigma_{x}{ }^{2}}\right] .
\end{aligned}
$$

Because collimators generally operate such that $x_{0} \geq$ $6 \sigma_{x}$ and $\Delta x<\sigma_{x}$, the difference of the error functions approaches zero, and the exponential terms approach one. So it is convenient to expand Eqs. (8) and (9) for small $\Delta x$. To lowest order in $\Delta x$ the average angle hitting the crystal is

$$
x_{p}^{\prime}=\left(x_{0}+\frac{\Delta x}{2}\right) \frac{\sigma_{x x^{\prime}}}{\sigma_{x}^{2}}
$$

To fifth order in $\Delta x$, the striking beam divergence becomes

$$
\begin{aligned}
\sigma_{x^{\prime}}\left(x_{0}\right)= & \frac{\sqrt{\epsilon\left(\epsilon+\langle\mathcal{H}\rangle \sigma_{p}^{2}\right)}}{\sigma_{x}}+\frac{\sigma_{x x^{\prime}}^{2} \Delta x^{2}}{24 \sigma_{x}^{3} \sqrt{\epsilon\left(\epsilon+\langle\mathcal{H}\rangle \sigma_{p}^{2}\right)}} \\
& \times\left\{1-\frac{\Delta x^{2}}{240 \sigma_{x}^{2}}\left[8+5 \frac{-\alpha \epsilon \sigma_{x x^{\prime}}+D^{2} D^{\prime 2} \sigma_{p}^{4}}{\epsilon\left(\epsilon+\langle\mathcal{H}\rangle \sigma_{p}{ }^{2}\right)}\right.\right. \\
& \left.\left.+12 \frac{x_{0}^{2}}{\sigma_{x}^{2}}\right]-\frac{x_{0} \Delta x^{3}}{20 \sigma_{x}^{4}}\right\}+O\left(\Delta x^{6}\right)
\end{aligned}
$$

where

$$
\langle\mathcal{H}\rangle=\gamma D^{2}+2 \alpha D D^{\prime}+\beta D^{\prime 2} .
$$

Higher order terms have a negligible contribution on $x_{p}^{\prime}$ and $\sigma_{x^{\prime}}\left(x_{0}\right)$ for typical RHIC parameters.

From Eqs. (10) and (11) and Fig. 1 it can be seen that the optimal location for a crystal collimator is a location where $\sigma_{x x^{\prime}}=0$. This untilts the phase ellipse. A location where $\alpha=D^{\prime}=0$ and $\beta$ is a local maximum flattens the phase ellipse and minimizes the beam divergence on the crystal. In addition, the angle and angular spread of the incident beam on the crystal is independent of $x_{0}$ which greatly simplifies the operation of a crystal collimator. Otherwise the crystal collimator needs to be realigned whenever its position is changed.

There are weaknesses in our model:

(i) The beam distribution is not necessarily Gaussian in the tails.

(ii) Changes in the distribution from multiple encounters with the crystal are not included.

(iii) Particles do not strike across the crystal face after the first few turns, which implies $\Delta x$ should be replaced by a parameter which scales as the mean impact parameter of particles on the crystal.

(iv) The actual beam distribution is truncated by apertures such as secondary collimators.

The mean angle of particles striking the crystal will be minimally affected by changes in these assumptions since it is a single particle effect. The calculated divergence is affected by changes in these assumptions, however, Eqs. (9) and (11) show the scaling of the divergence with the Twiss parameters. We will address these weaknesses in Sec. V.

\section{SIMULATION}

A Monte Carlo simulation, CATCH, was written to simulate channeling through a crystal lattice [13]. Figure 3 shows the result of a simulation using $\mathrm{CATCH}$. The left graph shows the horizontal particle phase space at the entrance of the crystal on the first turn of a simulation. The edges of the crystal and the range of angles that are within the acceptance of the crystal are shown. The right graph shows the horizontal phase space at the exit of the crystal after many turns. The channeled particles appear 

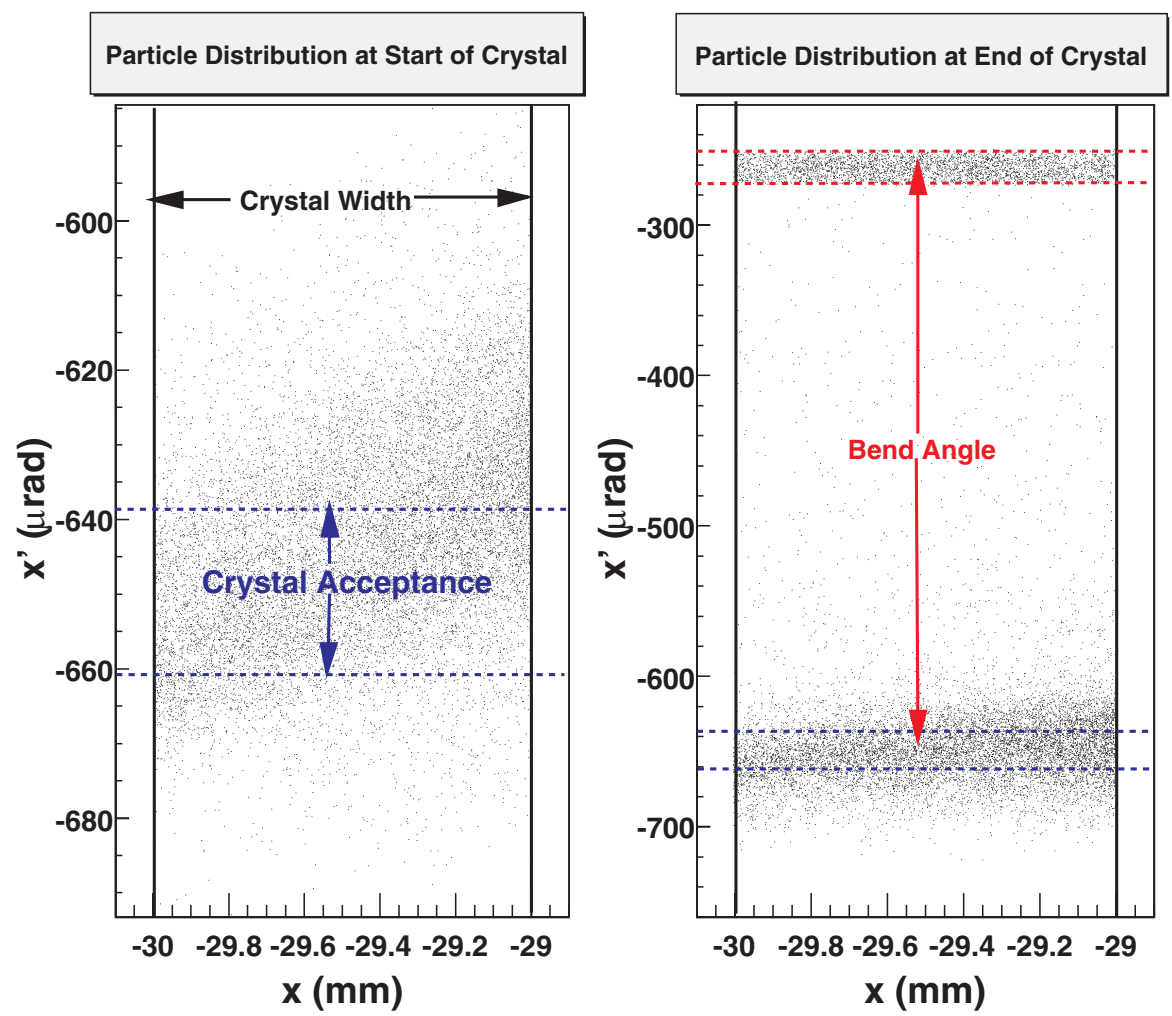

FIG. 3. (Color) Simulation of crystal channeling of particles hitting a silicon crystal. The left figure shows the horizontal phase space at the entrance to the crystal, indicating the crystal angular acceptance. The right figure shows the same phase space at the end of the crystal. The horizontal blue and horizontal red dashed lines indicate the angular acceptance and emittance of the crystal.

between the red dotted lines. They receive an angular kick approximately equal to the bend angle of $440 \mu \mathrm{rad}$. Particles that are not channeled, but scatter through the crystal appear at the bottom of the plot. Particles that channel first and then scatter out of the crystal do not get the full angular kick of the crystal. They appear at intermediate angles. In the vertical phase space, particles are tracked through the crystal as in a drift with additional scattering to simulate interactions with electrons within the crystal lattice.

The simulations used a $5 \mathrm{~mm}$ long, $5 \mathrm{~mm}$ high, $1 \mathrm{~mm}$ wide crystal. It had a bend angle of $440 \mu \mathrm{rad}$. These parameters match the crystal shown in Fig. 6 . The crystal has a smooth surface in the simulation.

A C ++ program was written, SCAN, that tracks particles around RHIC for a set number of turns and a range of crystal angles to simulate the effect of the crystal on the beam halo as the crystal angle is rotated [14]. SCAN uses a symplectic $6 \times 6$ matrix with no coupling or rf to track particles around RHIC. CATCH is used by SCAN to simulate particles striking the crystal.

The transverse particle distributions used in the simulation are identical to Eq. (5) with emittances of $15 \pi \mathrm{mm}$ mrad. The momentum offset distribution is a Gaussian with a fractional rms momentum spread of $0.13 \%$. The distributions are chosen with typical RHIC beam parameters. Particles that have a maximum horizon- tal position beyond the crystal, $-30.0 \mathrm{~mm}$ for Fig. 3, are removed from the distribution. The time difference coordinate between particles is chosen to be zero.

Figure 4 shows a SCAN simulation of the number of particles scattered from a crystal versus the crystal angle relative to the beam orbit. There are 40 turns in this simulation. A reduction in the scattering rate indicates channeling in the crystal. This is because channeling in the crystal deflects particles in a specific direction as

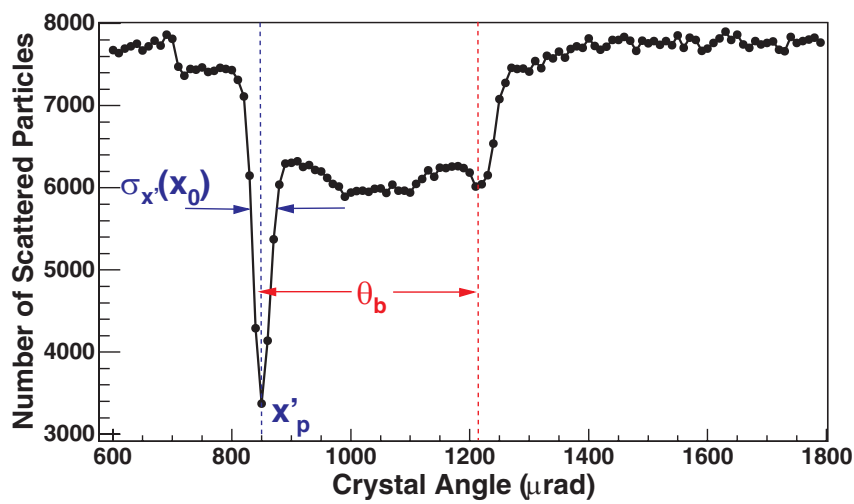

FIG. 4. (Color) Simulation of the number of scattered particles as the crystal angle is changed. A reduction in the scattering rate indicates channeling. The crystal is properly aligned at $x_{p}^{\prime}=$ $830 \mu \mathrm{rad}$. This is a different simulation than Fig. 3. 
opposed to scattering in a random direction. Channeled particles are removed from the population of particles that is likely to scatter into detectors such as a beam loss monitor. So for a given number of particles striking a crystal, reduced scattering indicated channeling.

The large thin dip occurs at an angle $\theta=830 \mu \mathrm{rad}$ where the crystal planes are aligned to the incoming particles as predicted by Eq. (10). The width of the dip is $10 \%$ wider than Eq. (11) predicts. This is due to the multiple turns in the simulation. Particles scatter through the crystal and return on a subsequent turn. These particles will add to the divergence of the beam that strikes the crystal, widening the dip. Volume capture occurs when the crystal angle is between 900 and $1200 \mu \mathrm{rad}$. At these angles, the particles are not aligned to the planes upon entering the crystal, but can scatter into the planes after traversing some distance in the crystal. The channeling stops at $\theta \approx$ $1275 \mu \mathrm{rad}$ because the planes are rotated too far for the particles to scatter into them.

The channeling efficiency, $\epsilon$, can be derived from the simulation by taking the ratio of reduction in scattering in the large dip to the scattering rate of particles with no channeling.

$$
\epsilon=1-\frac{N_{\min }}{N_{\max }}
$$

The efficiency obtained from Fig. 4 is $56 \pm 1 \%$, compared to the $52 \%$ predicted from Eq. (2).

\section{RHIC CRYSTAL COLLIMATOR}

Figure 5 shows a schematic of the RHIC crystal collimation system. The crystal collimator is located in the drift space of the yellow ring immediately downstream of the outgoing IR triplet for the PHENIX detector.

The crystal collimation system contains:

(i) The crystal collimator located in the "CC Vessel" in Fig. 5.

(ii) A $450 \mathrm{~mm}$ long inverted L-shaped dual plane copper scraper with a phosphor screen mounted on the front face to image channeled beam. (iii) A helium-neon (HeNe) laser and charge coupled device camera to measure the angle of the crystal relative to its line of motion.

(iv) A camera to image the phosphor screen on the yellow scraper (not shown).

(v) Two scintillators that form a hodoscope used to monitor particles scattered at large angles from the crystal.

(vi) Eight "upstream" PIN diodes between the crystal collimator and the scraper, used to detect particles scattered from the crystal.

(vii) An array of four "downstream" PIN diodes downstream of the scrapers, used to detect particles scattered from the scraper.

(viii) Four dedicated ionization chamber beam loss monitors to measure large beam losses downstream of the scraper.

(ix) A dual plane beam position monitor on each end of the collimation section to measure the beam position and angle at the crystal and scraper (not shown).

The crystal that is used in RHIC is an O-shaped silicon crystal, produced at PNPI, St. Petersburg, Russia, using the (110) planes for channeling. The crystal was mounted in the holder at IHEP, Protvino. It is shown in Fig. 6. The length of the side that channels the beam is $5 \mathrm{~mm}$. The width of the crystal is $\Delta x=1 \mathrm{~mm}$. The surface roughness is estimated to be $2 \mu \mathrm{m}$. The specified bending angle of the crystal is $\theta_{b} \approx 440 \mu \mathrm{rad}$. This bend is achieved with the stainless steel holder by applying inward pressure on the long sides of the crystal, forcing the short side to bulge outward. The beam enters the crystal from the top of Fig. 6, and the channeled beam is deflected to the right. The crystal was manufactured with a miscut angle of $\theta_{\text {miscut }}=$ $465 \mu \mathrm{rad}$. The miscut angle is the angle between the normal to the beam input face and the crystal planes. This type of crystal deflector was extensively used at IHEP for extraction with efficiency of about $40 \%$ [7].

The crystal sits at the end of a long lever arm mounted on a pivot that moves the crystal horizontally, transverse to the beam direction, via a translation stage and stepping motor. The crystal angle in the horizontal plane is changed by a piezoelectric inchworm. The inchworm pushes on the lever

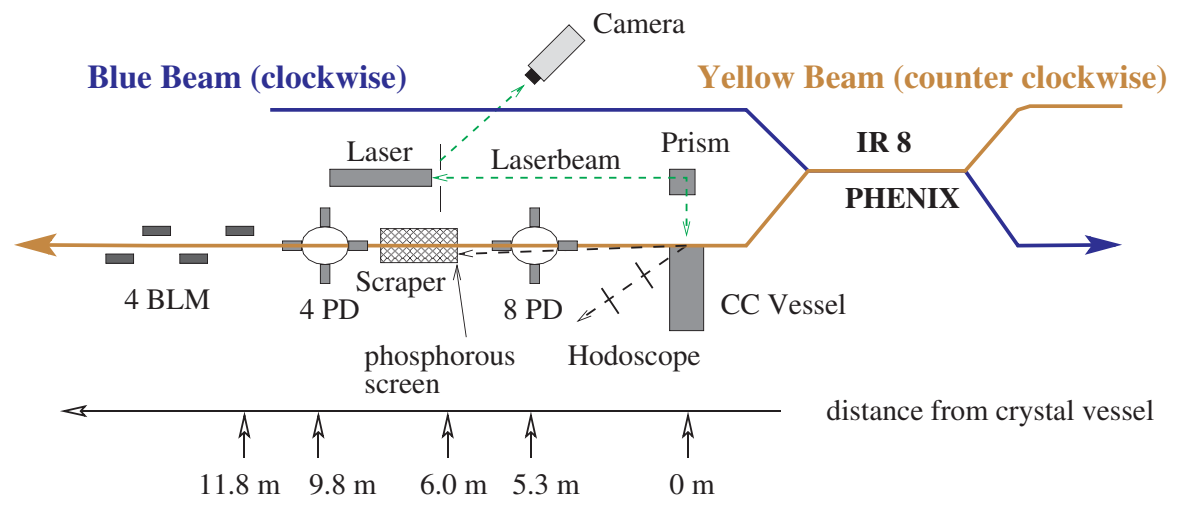

FIG. 5. (Color) RHIC crystal collimation system. The center of RHIC is toward the bottom of the figure. 


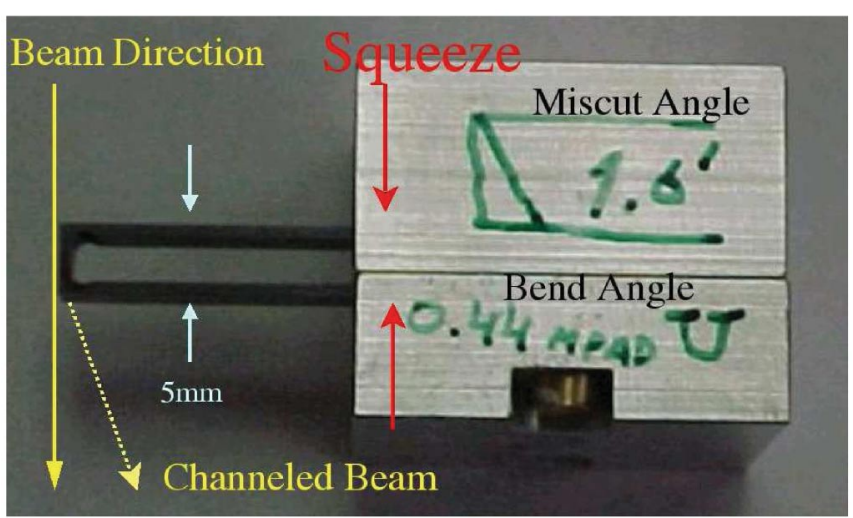

FIG. 6. (Color) The RHIC crystal. The RHIC beam enters the picture from the top. Channeled beam is diverted to the right.

arm causing the arm to pivot so as to change the angle of the crystal in the horizontal plane. The crystal angle is determined by reflecting the HeNe laser beam from the crystal face and measuring the deflection as the angle is changed.

Because the crystal is located in the interaction region matching section, any change in the $\beta^{*}$ at PHENIX significantly changes the Twiss parameters at the collimators. In addition, because of the placement of the dipole correctors, any steering done in PHENIX has an effect on the beam location and angle at the collimators. Figure 7 shows the model $\beta$ functions and dispersion through the collimation section in the yellow ring for $\beta_{\text {PHENIX }}^{*}=1 \mathrm{~m}$. Table I lists the model Twiss parameters at the location of the crystal for the various $\beta_{\text {PHENIX }}^{*}$

The beam in this section is converging so the divergence of the particles striking the crystal will be large. According
TABLE I. Design horizontal beam parameters at crystal for various $\beta_{\text {PHENIX }}^{*}$

\begin{tabular}{lrcrr}
\hline \hline$\beta_{\text {PHENIX }}^{*}[\mathrm{~m}]$ & $\beta[\mathrm{m}]$ & $\alpha$ & $D[\mathrm{~m}]$ & \multicolumn{1}{c}{$D^{\prime}$} \\
\hline 1 & 1155 & -26.5 & -0.864 & -0.0162 \\
2 & 581 & -12.8 & -0.152 & 0.0002 \\
3 & 391 & -8.33 & -0.013 & 0.0032 \\
5 & 242 & -4.95 & 0.103 & 0.0055 \\
\hline \hline
\end{tabular}

to Eqs. (10) and (11) this is not an optimal situation for two reasons. The first is that the angular divergence of particles hitting the crystal may be larger than the critical angle is the beam has a larger than normal emittance or energy spread. The second is that the optimal angle of the crystal will vary with the crystal position. More advantageous locations for placing the crystal collimator are excluded because they entail installing expensive cryogenic bypasses.

\section{CHANNELING RESULTS}

Data were taken during all RHIC runs between 2001 and 2003 with a variety of beam and optics combinations. Table II lists the existing data sets. Most of these data were taken during routine operations with minimal interference to the RHIC detectors. The term "scan" indicates that the crystal collimator was inserted to a transverse position such that scattering of the halo was detected on the upstream PIN diodes. The crystal is then rotated through a range of angles with respect to the beam orbit. During each scan, the beam loss rate was measured by the available detectors. The experimental background rates were also logged for a large number of the scans.
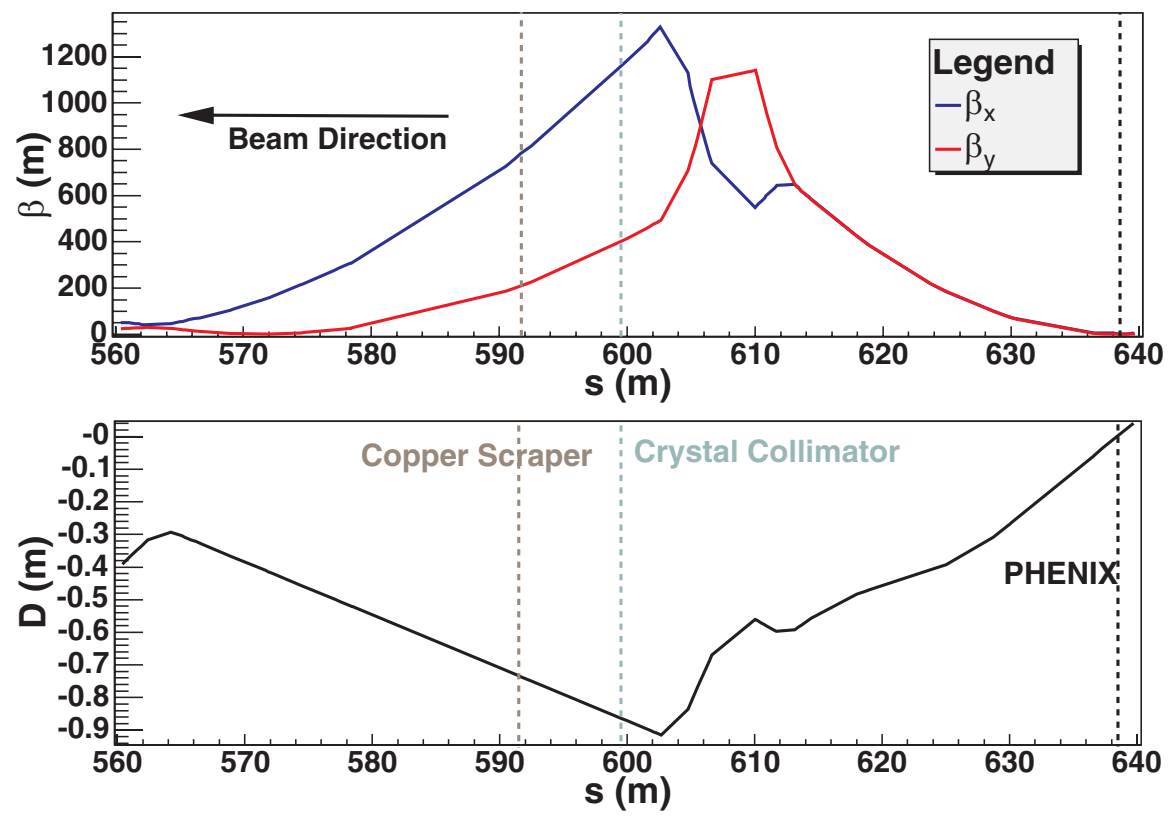

FIG. 7. (Color) Model horizontal $\beta$ functions and dispersion function near the crystal collimator for $\beta_{\mathrm{PHENIX}}^{*}=1 \mathrm{~m}$. 
TABLE II. Synopsis of channeling data.

\begin{tabular}{lcccc}
\hline \hline Run & Species & $\beta_{\text {PHENIX }}^{*}$ & No. of stores & No. of scans \\
\hline 2001 & $\mathrm{Au}$ & $5 \mathrm{~m}$ & 8 & 27 \\
2001 & $\mathrm{Au}$ & $2 \mathrm{~m}$ & 4 & 24 \\
2001 & $\mathrm{Au}$ & $1 \mathrm{~m}$ & 12 & 109 \\
2002 & $\mathrm{p}$ & $3 \mathrm{~m}$ & 11 & 119 \\
2003 & $\mathrm{Au}$ & $2 \mathrm{~m}$ & 4 & 20 \\
\hline \hline
\end{tabular}

Multiple scans were taken each store to vary parameters, such as the crystal position, within a single store.

Figure 8 is an example of a typical crystal scan using one of the upstream PIN diodes to monitor scattering from the crystal. The drop in the scattering rate indicates channeling in the crystal. The vertical error bars are statistical. The horizontal error bars are given by the resolution of the angular readback, which is approximately $25 \mu \mathrm{rad}$. Each scan was divided into $20 \mu \mathrm{rad}$ bins. A weighted average was computed for each bin to reduce fluctuations in the data. The averaged data were fit to

$$
f(\theta)= \begin{cases}A_{1}\left[1+\frac{\left(\theta-\theta_{1}\right)^{2}}{(8 \ln 2) w_{1}^{2}}\right]^{-1} & +S \theta+T: \theta \leq \theta_{A}, \\ A_{2} & +S \theta+T: \theta_{A}<\theta<\theta_{2}, \\ A_{2}\left[1+\frac{\left(\theta-\theta_{2}\right)^{2}}{(8 \ln 2) w_{2}^{2}}\right]^{-1} & +S \theta+T: \theta_{2} \leq \theta,\end{cases}
$$

where $\theta_{1,2}, w_{1,2}$, and $A_{1,2}$ are the centers, widths, and amplitudes of the left and right Lorentzian dips, $\theta_{A}$ is the end of the left Lorentzian, and $S$ and $T$ are the slope and offset of the background. The value of $\theta_{A}$ is determined by continuity of $f\left(\theta=\theta_{A}\right)$. Lorentzian distributions were chosen for fitting because initial fits with Gaussian distributions showed that the data contained non-Gaussian tails. Lorentzian distributions matched the data more closely.

It should be noted that $w_{1,2}$ are not the Lorentzian full widths at half maximum (FWHM) as is typically used for a

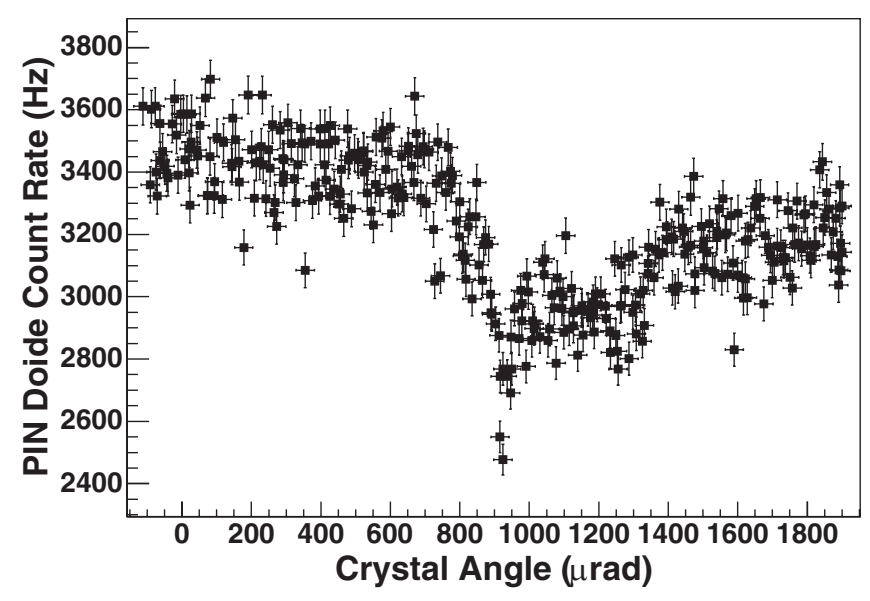

FIG. 8. Typical crystal scan during Fill 01737 with gold beam.
Lorentzian distribution [15]. Instead they are the rms of a Gaussian distribution with the equivalent FWHM. We did this to compare the width from these fits to Eq. (11) where a Gaussian distribution is used. Figure 9 shows the data from Fig. 8 after averaging. The fit is shown as a red line.

Each of the hodoscope scintillators and their coincidence signal were analyzed in the same fashion. However, the scintillators are subject to backgrounds coming from particles scraping in the interaction region triplet magnets immediately upstream of them. This reduced the signal to noise ratio and made it difficult to meaningfully fit the data. When such fits were possible, they are used. The downstream PIN diodes are not useful for this analysis because they are also sensitive to scattering from the copper scraper.

Many scans could not be used for a variety of reasons. Technical problems during a scan, such as a dipole corrector trip or magnet quench caused us to remove many scans from the analysis. No scans were eliminated based on agreement between the data and expectations from simulation or theory. Of the 301 total scans, approximately $40 \%$ could be analyzed.

Figure 10 shows a comparison between two simulations and data from the 2001 run. The blue curve uses the design Twiss parameters, the red curve is the simulation with measured optics. There are 20 turns in each simulation. They are scaled vertically to match the number of scattered particles in the simulations to the data. Each simulation is shifted horizontally to take into account the miscut angle and angular offset of the crystal. The shift amount is chosen arbitrarily to match the large dip in the measured optics simulation to the data. The same shift is used in each simulation.

The simulation using the design optics reproduces the general shape of the data. The measured optics more closely match the data. The shifted position in the channeling peak between the two simulations reflects a large change in $\sigma_{x x^{\prime}} / \sigma_{x}^{2}$ between the design and measured op-

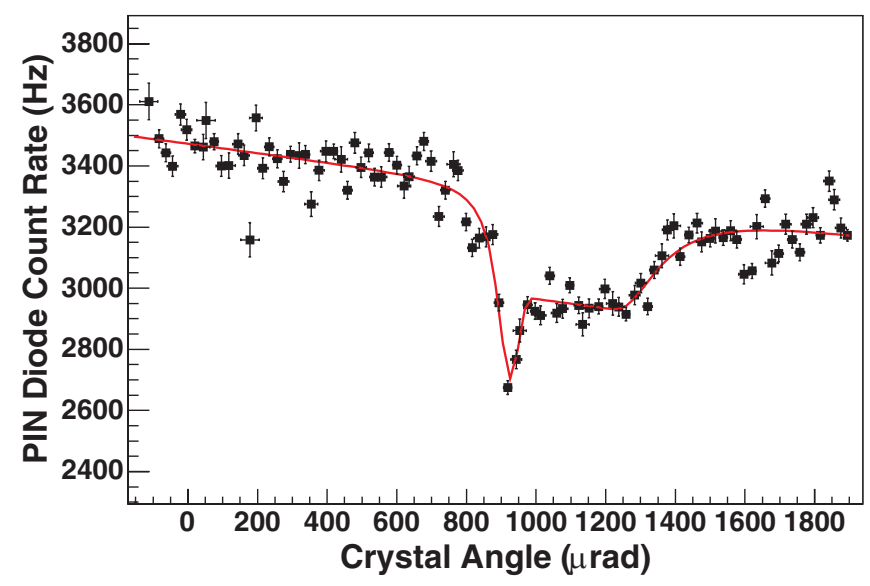

FIG. 9. (Color) Data from Fig. 8 after averaging. The red line corresponds to the fit to Eq. (14). 


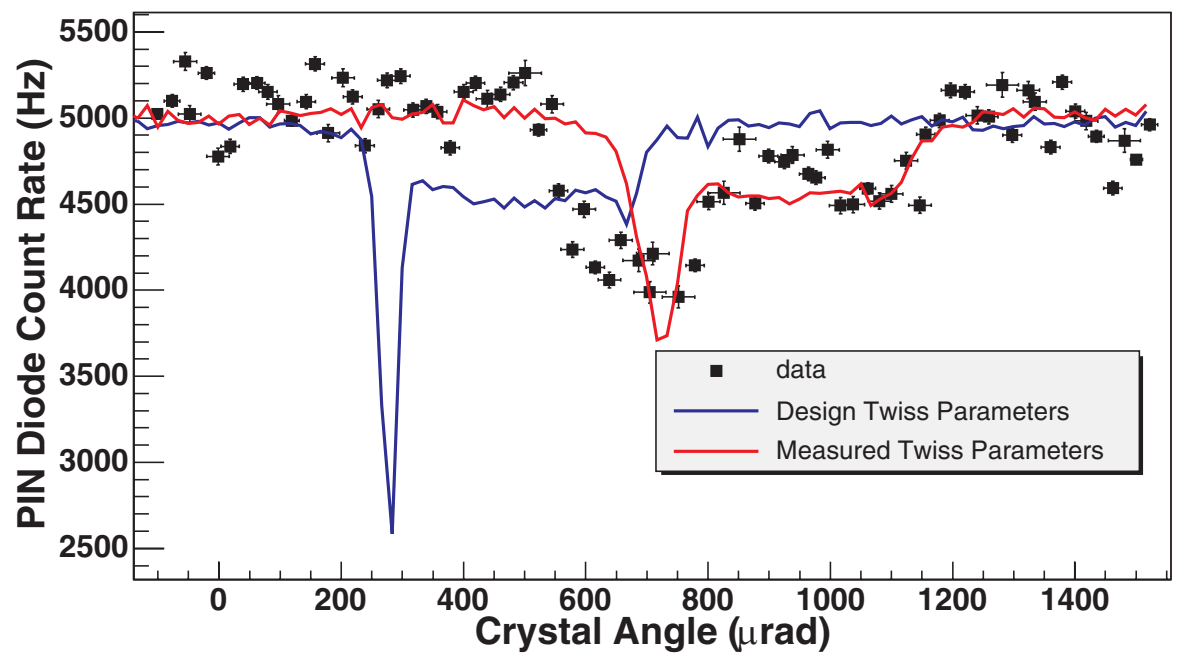

FIG. 10. (Color) Comparison of simulations with the data from Fill 1547. The distance between the crystal and beam is $29.0 \mathrm{~mm}$.

tics. Physically, $\sigma_{x x^{\prime}} / \sigma_{x}^{2}$ is the tilt of the horizontal phase ellipse, which is greater in the measured optics than the design.

The width is 5 times narrower than the data show for the design optics, and 3.2 times narrower for the measured optics. The difference between the two simulations is due to the increased tilt of the phase ellipse in the measured optics, leading to a larger angular divergence striking the crystal.

The efficiency, $\epsilon$, is approximately 2.6 times larger than the channeling data for the design optics. The measured Twiss parameters show better agreement with the channeling data, being only 1.5 times larger. The differences in efficiency between the two simulations are from the differences in the channeling width just discussed. The larger angular divergence from more tilted phase ellipse leads to a lower efficiency.

Measuring the channeling angle as a function of the distance between the crystal and the beam, $x_{0}$, is a way to measure the slope of the phase ellipse, $\sigma_{x x^{\prime}} / \sigma_{x}^{2}$. This is shown in Fig. 11 for all of the $\beta_{\text {PHENIX }}^{*}=1 \mathrm{~m}$ data. The data are fit to a line whose slope is given by $\sigma_{x x^{\prime}} / \sigma_{x}^{2}$ as in Eq. (11). The error bars are statistical. Points with $x_{0}>$ $37 \mathrm{~mm}$ have lower angles than predicted by the model. At positions in this range, the crystal is very much in the beam halo and the signal to noise ratio is low. Nevertheless, the points are included in the fit. Removing them from the fit increases the fit value of $\sigma_{x x^{\prime}} / \sigma_{x}^{2}$ by $2 \%$ which is well within the error bars.

Table III shows the results for the available data. The measured $\beta_{\text {PHENIX }}^{*}=1 \mathrm{~m}$ optics do not agree with the measured $\sigma_{x x^{\prime}} / \sigma_{x}^{2}$ from the channeling data. Table III shows that the design value of $\sigma_{x x^{\prime}} / \sigma_{x}^{2}$ only varies within $10 \%$ when $\beta_{\text {PHENIX }}^{*}$ is changed. This is expected for the measured optics as well. The $\sigma_{x x^{\prime}} / \sigma_{x}^{2}$ of the channeling data in the $\beta_{\text {PHENIX }}^{*}=1 \mathrm{~m}$ case agrees with measured values of $\sigma_{x x^{\prime}} / \sigma_{x}^{2}$ for $\beta_{\text {PHENIX }}^{*}=2 \mathrm{~m}$. This indicates that the measured Twiss parameters for $\beta_{\mathrm{PHENIX}}^{*}=1 \mathrm{~m}$ might be wrong. However, even careful checking of the measurement procedure and the data did not reveal any obvious problem and the reason for this disagreement remains unknown.

The predicted and average beam divergence, $\sigma_{x^{\prime}}\left(x_{0}\right)$, is listed in Table IV for each $\beta^{*}$. Corrections to $\sigma_{x^{\prime}}\left(x_{0}\right)$ from $x_{0}$ are less than $1 \mu \mathrm{rad}$ for all used RHIC parameters and crystal positions so the average is performed over all $x_{0}$.

The width of the channeling dip is $2-3$ times larger than expected from both the model and simulations. Simulations provide widths that are slightly larger than Eq. (11) predicts. The size of this difference is less than $10 \%$ for 40 turn simulations and between $30 \%$ and $80 \%$ for simulations with 80 turns. This increase in width comes from particles that encounter the crystal multiple times.

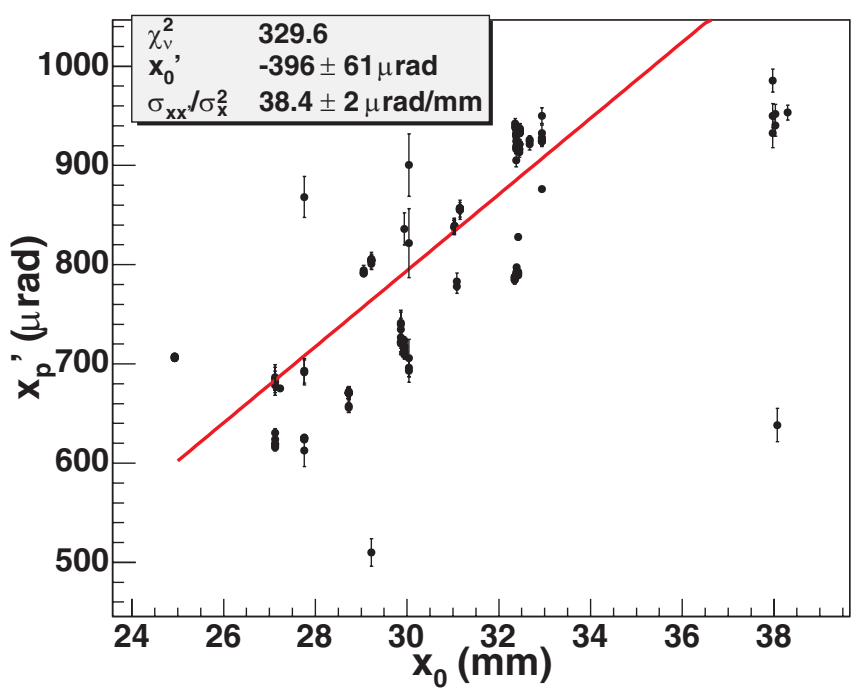

FIG. 11. (Color) Graph of the channeling dip vs crystal location for $\beta_{\text {PHENIX }}^{*}=1 \mathrm{~m}$. All points are included in the fit. 
TABLE III. Comparison of measured $\sigma_{x x^{\prime}} / \sigma_{x}^{2}$ to model.

\begin{tabular}{lcccc}
\hline \hline Run & $\beta_{\text {PHENIX }}^{*}[\mathrm{~m}]$ & Design optics & $\begin{array}{c}\sigma_{x x^{\prime}} / \sigma_{x}^{2}[\mu \mathrm{rad} / \mathrm{mm}] \\
\text { Measured optics }\end{array}$ & Channeling data \\
\hline 2001 & 5 & 21 & $\ldots$ & $\ldots$ \\
2001 & 2 & 22 & $37 \pm 2$ & $\ldots$ \\
2001 & 1 & 23 & $23 \pm 3$ & $38 \pm 2$ \\
2002 & 3 & 21 & $\ldots$ & $\ldots$ \\
2003 & 2 & 22 & $36 \pm 2$ & $36 \pm 2$ \\
\hline \hline
\end{tabular}

TABLE IV. Comparison of various predictions of $\sigma_{x^{\prime}}\left(x_{0}\right)$.

\begin{tabular}{lccccc}
\hline \hline \multirow{2}{*}{ Run } & $\beta^{*}[\mathrm{~m}]$ & Model optics & Measured optics & Simulation & Channeling data \\
\hline 2001 & 5 & 12.3 & $\ldots$ & $\ldots$ & $39 \pm 4$ \\
2001 & 2 & 9.98 & $19 \pm 1$ & $20 \pm 1$ & $78 \pm 4$ \\
2001 & 1 & 8.91 & $9 \pm 1$ & $11 \pm 1$ & $35 \pm 3$ \\
2002 & 3 & 10.8 & $\ldots$ & $\ldots$ & $58 \pm 3$ \\
2003 & 2 & 9.98 & $14 \pm 1$ & $16 \pm 1$ & $28 \pm 2$ \\
\hline \hline
\end{tabular}

This is not included in Eq. (11). The number of turns also affects the depth of the volume capture region which must be matched to the data as Fig. 12 shows [14]. This sets a limit on the number of turns in the simulation, ranging from 20 to 80, which in most cases is not enough to explain the whole difference. The simple model used for the simulation is not sophisticated enough to model realistic survival times of particles. Other processes that may change the particle angles are not included, nor are realistic machine apertures. However, the longer simulation allows a scattered particle more chances to strike the crystal and perhaps be channeled on subsequent turns.

The values of the rms momentum spread, $\sigma_{p}$, and emittance used for the calculations and simulations are as- sumed to be the nominal RHIC parameters listed in Table IV. Variations in the emittance have little effect on the width of the channeling dip for typical RHIC emittances. For RHIC parameters,

$$
\sigma_{x^{\prime}}\left(x_{0}\right) \propto 1[\mu \mathrm{rad}] \sigma_{p} .
$$

This linearity continues until $\sigma_{p} \approx 7 \sigma_{p \text {,nom. }}$. However, it is not possible for the rms momentum spread to be more than a factor of 2 from the nominal value unless there is a large fraction of the beam outside of the rf bucket [16]. This was never the case during crystal studies and is not a cause of the increased $\sigma_{x^{\prime}}\left(x_{0}\right)$ in the channeling data.

Beam profile measurements using the scrapers show that the beam tails are broader than a Gaussian distribution

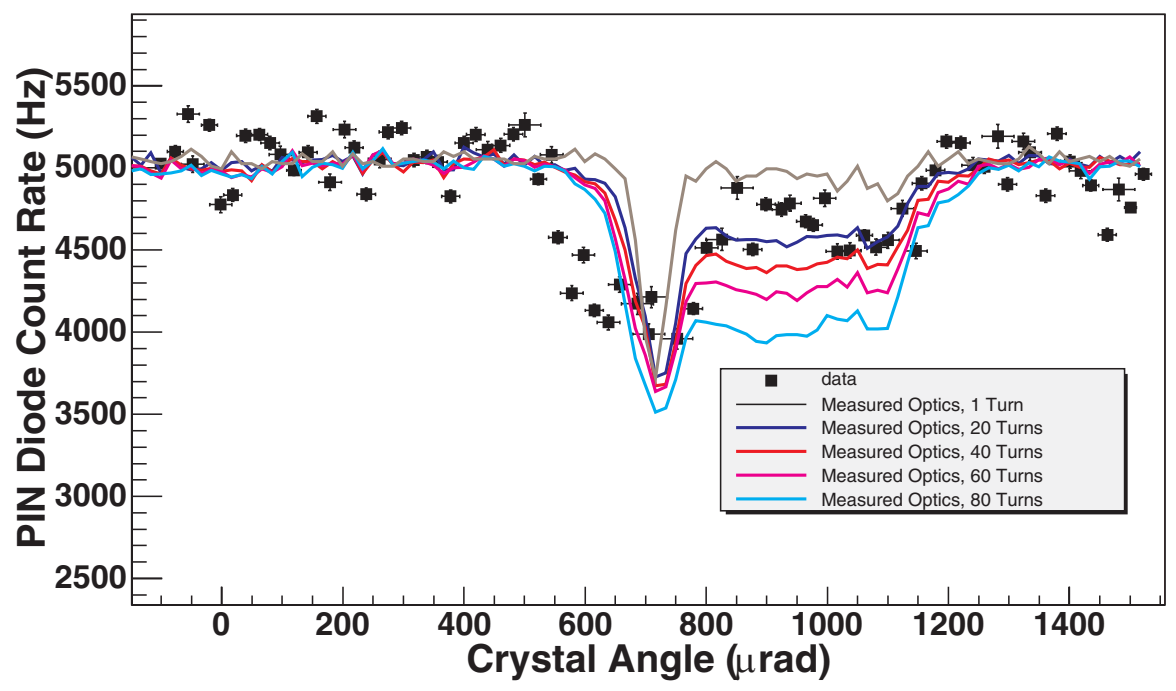

FIG. 12. (Color) Comparison of various simulations with the data from Fill 1547. The depth of the volume capture region increases as the number of turns in the simulations increase. 
$[14,17]$. This partially accounts for the increased width of the channeling peak. The non-Gaussian tails change the shape of the angular distribution of particles that hit the crystal. The change of the halo distribution will have a large effect on $\sigma_{x^{\prime}}\left(x_{0}\right)$, even though the possible range of angles which strike the crystal is unchanged.

There is a noticeable discrepancy in $\sigma_{x^{\prime}}\left(x_{0}\right)$ between the two data sets that have $\beta_{\text {PHENIX }}^{*}=2 \mathrm{~m}$. The average width in the 2001 run is 2.8 times larger than the average width from 2003 run. Differences in the Twiss parameters predict only a $25 \%$ difference between the data sets. A larger momentum spread from debunched beam could explain the increase. In the worst case only $25 \%$ of the beam was debunched, and would need to increase the rms momentum spread to 2.1 times larger than the nominal value to explain the increased width. This is not likely to be the case and so cannot explain the effect. The increased channeling width for the $2001 \beta_{\text {PHENIX }}^{*}=2 \mathrm{~m}$ data is not understood.

The channeling efficiency is determined by dividing the depth of the channeling dip by the background rate. Table V shows the expected channeling efficiency, comparing calculations using design optics, measured optics, simulations, and the average measured channeling efficiency. Simulations agree with the theory when using the measured optics.

The measured efficiency does not match any theory or simulation. This disagreement stems from the disagreement in the value of $\sigma_{x^{\prime}}\left(x_{0}\right)$. All other parameters used for the calculation are a function of the crystal properties or the beam energy. If the measured value of $\sigma_{x^{\prime}}\left(x_{0}\right)$ is used to calculate the channeling efficiency, the agreement is within $20 \%$ for four of the runs. The measured efficiency of the $\beta_{\mathrm{PHENIX}}^{*}=2 \mathrm{~m}$ data in the 2001 run is 3 times larger than the expected efficiency using the measured channeling width. It is, however, consistent with the measured efficiency in the 2003 data with $\beta_{\text {PHENIX }}^{*}=2 \mathrm{~m}$. This and Table IV indicate that there is an underlying problem with the $\sigma_{x^{\prime}}\left(x_{0}\right)$ measurement in the 2001 data set. Nevertheless, the agreement between the measured channeling efficiency and measured width of the channeling dip shows that the physics of crystal channeling is, in general, understood. This analysis shows that proper understanding of $\sigma_{x^{\prime}}\left(x_{0}\right)$ is essential to predicting the channeling efficiency correctly.
The low channeling efficiency from the large $\sigma_{x^{\prime}}\left(x_{0}\right)$ masked effects due to imperfect channeling at the edge of the crystal. These could possibly be investigated in situations with higher channeling efficiencies.

Measurements at the SPS extracting $\mathrm{Pb}^{82+}$ at $106 \mathrm{GeV} / u / c$ show efficiencies of $(8-14) \%$ in agreement with their predictions [3]. Similar measurements with protons at $120 \mathrm{GeV}$ show an extraction efficiency of $(15.1 \pm$ $1.2) \%$, in agreement with predictions [6]. For these measurements, the beam is excited using a horizontal stochastic kick. In these measurements the efficiency is calculated by measuring the ratio of the extracted $\mathrm{Pb}$ ion current to the lost beam current, and suffers from large errors due to uncertainties in the beam lifetime.

Similar extraction experiments were performed at the Tevatron using bent crystals [5]. Channeling efficiencies of $(35 \pm 11) \%$ were obtained during luminosity driven proton extraction, in agreement with simulation and Eq. (2) using the stated beam parameters [18]. Phosphor screens in the extraction line show dechanneling tails that are akin to our volume capture region.

In these experiments, the channeled beam was extracted into an independent beam line in which direct measurements on the extracted beam could be performed. Extraction efficiencies were calculated by measuring the increased beam loss when the crystal was inserted into the beam. In our case, we have no ability to perform direct diagnostics on the channeled beam. Although a phosphor screen is mounted on the front of the scraper, the imaging camera was damaged by radiation before any meaningful data could be obtained.

The Tevatron experiment deflected the beam in the vertical plane while inserting the crystal in the horizontal. This feature allowed the crystal to sample the entire vertical phase space. This makes the experiment insensitive to the halo distribution in the vertical plane because less than $1 \%$ of the vertical phase space lies outside of $3 \sigma_{y^{\prime}}$. The halo distribution in the horizontal plane, the plane in which the scraping occurs, does not have an effect on the channeling efficiency, although it affects such things as impact parameters. Our experiment is sensitive to the horizontal halo distribution because the crystal bends in the same plane as it scrapes. This causes the crystal to explicitly sample the horizontal angular divergence of the horizontal

TABLE V. Comparison among various models of expected channeling efficiency.

\begin{tabular}{lcccccc}
\hline \hline & & & \multicolumn{2}{c}{ Calculated channeling efficiency } & \multicolumn{2}{c}{ Measured } \\
Run & $\beta_{\text {PHENIX }}^{*}[\mathrm{~m}]$ & Design optics & Measured optics & Simulation & Measured width & Channeling efficiency \\
\hline 2001 & 5 & $59 \%$ & $\ldots$ & $\ldots$ & $(19 \pm 2) \%$ & $(24 \pm 3) \%$ \\
2001 & 2 & $71 \%$ & $(39 \pm 2) \%$ & $(37 \pm 1) \%$ & $(9 \pm 1) \%$ & $(28 \pm 3) \%$ \\
2001 & 1 & $74 \%$ & $(75 \pm 1) \%$ & $(56 \pm 3) \%$ & $(20 \pm 2) \%$ & $(19 \pm 3) \%$ \\
2002 & 3 & $79 \%$ & $\ldots$ & $\ldots$ & $(21 \pm 1) \%$ & $(26 \pm 3) \%$ \\
2003 & 2 & $71 \%$ & $(52 \pm 2) \%$ & $(50 \pm 1) \%$ & $(26 \pm 2) \%$ & $(26 \pm 3) \%$ \\
\hline \hline
\end{tabular}


halo which is scraped. The vertical distribution of the beam has no bearing on the channeling efficiency in our experiment to first approximation.

Our efficiencies are higher than those measured at the SPS, but lower than those in the Tevatron and IHEP. Silicon crystals were used in all cases with similar beam rigidities. The major cause of differences in the efficiencies is most likely the different beam divergences in each case.

\section{CRYSTAL COLLIMATION}

The bent crystal collimator was to used as part of a two stage collimation system. The first stage of a conventional two stage system acts as a scatterer for the beam halo, which is to be intercepted by multiple secondary collimators [2]. The purpose of the bent crystal was to act as a first stage collimator which directs the beam halo into a secondary collimator rather than merely scattering the halo, thereby improving the collimation efficiency. Another benefit is reducing the number of secondary collimators that are needed which would simplify the operation of the entire collimation system.

Once the crystal collimator is properly aligned to the beam halo, the copper scraper must be positioned to intercept the channeled particles. If the channeling efficiency is low, as in the case of RHIC, the scraper must also intercept the particles that scatter from the crystal.

Besides equipment protection, the ultimate purpose of a collimation system in RHIC is to reduce backgrounds in the experiments. Various background signals were recorded from each experiment to measure the effectiveness of the crystal collimator in removing these backgrounds. The STAR detector is located directly downstream of the crystal collimator and is therefore most sensitive to its effects.

These measurements were conducted during four stores using gold and proton beams. The crystal collimator and copper scraper were positioned in the beam halo. The crystal angle would be scanned to align the crystal to the beam to maximize channeling. The scraper would then be moved relative to the crystal. For three of the stores, the crystal was scanned again to measure the effect of crystal angle on the STAR background. For the remaining store, the crystal remained in the aligned position. The crystal and scraper would then be inserted further. Several times, the crystal and scraper were retracted to measure the STAR background without any collimation.

Figure 13 shows the ratio of the STAR background with the crystal channeling to the uncollimated background as a function of the difference of the normalized positions of the crystal and the scraper. A negative relative position indicates that the crystal is the primary aperture. At zero, the crystal and scraper are at the same betatron amplitude. As the scraper comes closer to the beam, the STAR background decreases, but rarely drops below the level of the uncollimated background indicated by the red dotted line.

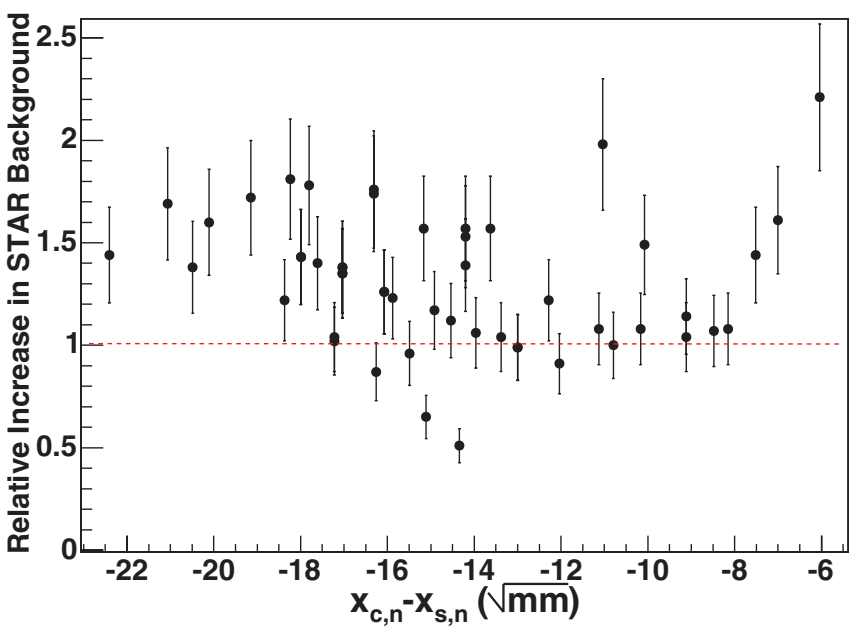

FIG. 13. (Color) The relative increase of the STAR background vs the relative normalized position between the crystal and the scraper during Fill 03061. The red line indicates where the crystal collimated background equals the collimated background. The error bars are statistical.

The few times that crystal collimation is successful, shown by points below the dotted red line, are not understood. These points are taken within a 5 min interval, along with other points. There is no a priori reason that these points should be more successful than the others. Nothing special occurred during these data points to make collimation more effective [14].

The reason that the crystal does not act as a good collimator is the low channeling efficiency. The large amount of scattering caused by the crystal cannot be removed with the single scraper. More detailed analysis of collimation results can be found in Refs. [14,19].

Adjusting the Twiss parameters to more optimal values as discussed in Sec. II can only be done by relocating the crystal collimator. All warm sections in RHIC have similar Twiss parameters. Placing the crystal in a cold region with more favorable Twiss parameters would require expensive cryogenic bypasses. More secondary collimators may alleviate the problem. These secondary collimators would intercept the scattered particles from the crystal collimator. However, the crystal collimator acts no differently than an ordinary primary collimator in this case.

\section{CONCLUSION}

We have demonstrated bent crystal channeling in the RHIC yellow ring using a bent silicon crystal during the 2001 through 2003 runs with polarized protons and fully stripped gold ions.

We developed an analytical model and used Monte Carlo simulations to estimate the channeling efficiency. These tools allow us to compute the change in crystal alignment as a function of the distance between the crystal and the beam core, the divergence of the beam that strikes the crystal, and channeling efficiency of the crystal. 
Measurements of $\sigma_{x x^{\prime}} / \sigma_{x}^{2}$ show an increase of $66 \%$ compared to predictions using the design optics. This is traced back to errors in the Twiss parameters at the crystal collimator.

The divergence of the beam that strikes the crystal is approximately 3-4 times larger than predicted by our model. Errors in the Twiss parameters cannot account for this discrepancy alone. Our model assumes that the beam has a Gaussian distribution while profile measurements with the RHIC scrapers show non-Gaussian tails.

The predicted channeling efficiency is 4 times larger than the measured values. The predicted efficiency relies on the assumed beam divergence hitting the crystal. If the measured beam divergence is used to the predict channeling efficiency, the agreement is better than $20 \%$ in all but one case.

We conclude that accurate knowledge of the transverse phase space is necessary when predicting the efficiency of crystal channeling, particularly if the crystal bends in the same plane as it scrapes. Collimation using the bent crystal was unsuccessful because of the low channeling efficiency. More particles were scattered from the crystal than were channeled. One secondary absorber was not enough to intercept all of the particles coming from the crystal.

The crystal collimator was removed after the 2003 run and the RHIC collimation system was upgraded because of the unsatisfactory performance of the crystal collimator. Details of the upgrade and experience with the new system are discussed in Refs. [14,20].

[1] R. Aßmann, E. B. Holzer, J. B. Jeanneret, V. Kain, S. Redaelli, G. Robert-Demolaize, and J. Wenninger, in Proceedings of the 2004 European Particle Accelerator Conference, Lucerne, Switzerland (CERN, Geneva, Switzerland, 2004).

[2] T. Trenkler and J. B. Jeanneret, CERN SL Note No. 95-03, LHC 312, 1995.

[3] G. Arduini, C. Biino, M. Clément, K. Cornelis, N. Doble, K. Elsener, G. Ferioli, G. Fidecaro, L. Gatignon, P. Grafström, M. Gyr, W. Herr, J. Klem, U. Mikkelsen, E. Weisse, S. P. Møller, E. Uggerhøj, A. Taratin, A. Freund, P. Keppler, and J. Major, Phys. Rev. Lett. 79, 4182 (1997).

[4] Jukka Klem, Helsinki Institute of Physics Internal Report No. 1998-02, 1998.

[5] R. A. Carrigan Jr., D. Chen, G. Jackson, N. Mokhov, C. T. Murphy, S.I . Baker, S. A. Bogacz, D. Cline, S. Ramachandran, J. Rhoades, J. Rosenzweig, A. Aseev and V. Biryukov, A. Taratin, J. A. Ellison, A. Khanzadeev, Y. Prokofieva, V. Samsonov, G. Solodov, B. Newberger, E. Tsyganov, H.-J. Shih ,W. Gabella, B. Cox, V. Golovatyuk, and A. McManus, Phys. Rev. ST Accel. Beams 1, 022801 (1998); R. A. Carrigan Jr., D. Chen, G. Jackson, N. Mokhov, C. T. Murphy, S.I. Baker, S. A. Bogacz, D.
Cline, S. Ramachandran, J. Rhoades, J. Rosenzweig, A. Aseev and V. Biryukov, A. Taratin, J.A. Ellison, A. Khanzadeev, Y. Prokofieva, V. Samsonov, G. Solodov, B. Newberger, E. Tsyganov, H.-J. Shih ,W. Gabella, B. Cox, V. Golovatyuk, A. McManus, Phys. Rev. ST Accel. Beams 5, 043501 (2002).

[6] G. Arduini, K. Elsener, G. Fidecaro, M .Gyr, W. Herr, J. Klem, U. Mikkelsen, and E. Weisse, in Proceedings of the 1997 Particle Accelerator Conference, Vancouver, BC, Canada (IEEE, Piscataway, NJ, 1998).

[7] A. G. Afonin, A. A. Arkhipenko, V. T. Baranov, V. M. Biryukov, Yu. A. Chesnokov, V. A. Gavrilushkin, V. N. Gres, V.I. Kotov, A. V. Minchenko, V.I. Terekhov, E. F. Troyanov, V.A. Zelenov, B.A. Chunin, A. S. Denisov, M. G. Gordeeva, Yu. M. Ivanov, A. A. Petrunin, and V. V. Skorobogatov, Phys. Lett. B 435, 240 (1998); A. G. Afonin, V.T. Baranov, V.M. Biryukov, M. B.H. Breese, V.N. Chepegin, Yu. A. Chesnokov, V. Guidi, Yu. M. Ivanov, V. I. Kotov, G. Martinelli, W. Scandale, M. Stefancich, V.I. Terekhov, D. Trbojevic, E. F. Troyanov, and D. Vincenzi, Phys. Rev. Lett. 87, 094802 (2001); A. G. Afonin et al., Instrum. Exp. Tech. 45, 476 (2002).

[8] D. Trbojevic, V. Biryukov, M. Harrison, B. Parker, P. Thompson, A. Stevens, N. Mokhov, and A. Drozhdin, in Proceedings of the 1998 European Particle Accelerator Conference, Stockholm, Sweden (IOP, London, 1998).

[9] J. Lindhard, K. Dan. Vidensk. Selsk. Mat. Fys. Medd. 34, 1 (1965).

[10] V. M. Biryukov, Yu. A. Chesnokov, and V.I. Kotov, Crystal Channeling and Its Application at High Energy Accelerators (Springer-Verlag, Berlin, Heidelburg, 1997).

[11] James A. Ellison, Theory of Particle Motion in Straight and Distorted Crystals in Relativistic Channeling edited by Richard A. Carrigan Jr. and James A. Ellison, NATO ASI, Ser. B, Vol. 165 (Plenum Press, New York, 1987).

[12] W. Feller, An Introduction to Probability Theory and Its Applications (John Wiley \& Sons, Inc., New York, 1971), 2nd ed., Vol. II.

[13] V. Biryukov, CERN SL Note 93-74 (AP), 1993. See also [10].

[14] R.P. Fliller III, Ph.D. thesis, Stony Brook, 2004; Brookhaven National Laboratory C-A/AP Note 170, 2004.

[15] Philip R. Bevington and D. Keith Robinson, Data Reduction and Error Analysis for the Physical Sciences (McGraw-Hill, Inc., New York, 1992), 2nd ed.

[16] Michael Blaskiewicz (private communication).

[17] R. Fliller III and A. Drees, Brookhaven National Laboratory C-A/AP Note 132, 2004.

[18] V. Biryukov, Phys. Rev. E 52, 2045 (1995).

[19] R. P. Fliller III, A. Drees, D. Gassner, L. Hammons, G. McIntyre, S. Peggs, D. Trbojevic, V. Biryukov, Y. Chesnokov, and V. Terekhov, Nucl. Instrum. Methods Phys. Res., Sect. B 234, 47 (2005).

[20] A. Drees, R. Fliller, and W. Fu, in Proceedings of the 33rd ICFA-HB2004 Workshop, Bensheim, Germany, AIP Conf. Proc. No. 773 (AIP, New York, 2004). 